\title{
TRANSVERSE KNOTS AND KHOVANOV HOMOLOGY
}

\author{
Olga Plamenevskaya
}

\begin{abstract}
We define an invariant of transverse links in $\left(S^{3}, \xi_{s t d}\right)$ as a distinguished element of the Khovanov homology of the link. The quantum grading of this invariant is the self-linking number of the link. For knots, this gives a bound on the self-linking number in terms of Rasmussen's invariant $s(K)$. We prove that our invariant vanishes for transverse knot stabilizations, and that it is non-zero for quasipositive braids. We also discuss a connection to Heegaard Floer invariants.
\end{abstract}

\section{Introduction}

1.1. Legendrian and transverse knots. There are two important classes of knots in a contact 3 -sphere $\left(S^{3}, \xi_{\text {std }}\right)$ : Legendrian knots and transverse knots. Legendrian knots are everywhere tangent to the contact planes; transverse knots are everywhere transverse to them. There are simple "classical" invariants for both classes: the Thurston-Bennequin and the rotation number for Legendrian knots, and the selflinking number for transverse knots. While certain knot types, e.g. all torus knots [EH1], are completely classified by these invariants (in this case the knot type is said to be Legendrian resp. transversely simple), for most knot types the classification is not known. There exist smoothly isotopic Legendrian resp. transverse knots with the same classical invariants which are not isotopic through Legendrian resp. transverse knots. Legendrian knots are somewhat better understood and enjoy a rich theory in the context of contact homology and symplectic field theory [Ch], [Ng], [EGH]: a differential graded algebra associated to a knot yields new Legendrian knot invariants. Transverse non-simplicity of certain knot types was demonstrated by Birman and Menasco in [BM] and by Etnyre and Honda in [EH3]. However, the existing examples are sparse (those of [EH3] are not even explicit), and the proofs in [BM] and [EH3] require a subtle analysis of specific braids and contact manifolds. Unlike Legendrian knots, transverse knots do not have any known efficient non-classical invariants.

1.2. The invariant $\psi(L)$. In this paper we introduce a transverse link invariant $\psi(L)$ as a distinguished element of the Khovanov homology of $L$. Given a closed braid diagram representing the transverse link, $\psi(L)$ is defined via a certain resolution of $L$. This invariant encodes the self-linking number: the quantum degree of $\psi(L)$ is given by $s l(L)$. It also discerns transverse stabilizations: if $L$ arises as a transverse stabilization of another transverse link, $\psi(L)$ vanishes. On the other hand, $\psi(L) \neq$ 0 for quasipositive braids. While we don't have any examples of transverse knots distinguished by $\psi(L)$ but not $s l(L)$ (indeed, we show that the invariant is the same for the pairs of transversely non-isotopic knots from $[\mathrm{BM}]$ ), we hope that a connection to Khovanov homology might be helpful. In particular, we establish a bound on the

Received by the editors March 7, 2005. 
self-linking number of a transverse knot in terms of the Khovanov homology knot invariant of Rasmussen [Ra].

1.3. Khovanov homology and low-dimensional topology. Khovanov homology is an invariant of knots and links introduced in [Kh]. Given a link $L$ in $S^{3}, K h(L)$ is a graded homology module whose graded Euler characteristic is the unnormalized Jones polynomial of $L$. As was recently discovered, the Khovanov homology has an interesting relation to low-dimensional topology. Ozsváth and Szabó [OS2] construct a spectral sequence converging to the Heegaard Floer homology $\widehat{H F}(-Y)$ of the double cover $Y$ of $S^{3}$ branched over a link $L$; the $E^{2}$ term of this spectral sequence is the (reduced) Khovanov homology of the link L. Rasmussen [Ra] uses Khovanov homology to give a combinatorial proof of the Milnor conjecture (i.e., to determine the slice genus of a torus knot). Our transverse link invariant suggests a further connection to contact topology. In fact, we can define a similar invariant in the reduced Khovanov theory; it is plausible that this invariant "corresponds" to the Ozsváth-Szabó contact invariant of the double cover of $\left(S^{3}, \xi_{\text {std }}\right)$ branched over the transverse link under the spectral sequence of [OS2].

\section{Preliminaries on transverse knots}

We collect a few necessary facts about transverse knots and their relation to braids here, referring the reader to [Et] for a detailed survey.

It will be convenient to work with closed braid representations of transverse knots. Consider $S^{3}$ equipped with the (rotationally symmetric) standard contact structure $\xi_{\text {std }}=\operatorname{ker}(d z-y d x+x d y)$. It easy to see that any closed braid around the $z$-axis can be made transverse to the contact planes. Moreover, by a theorem of Bennequin [Be] any transverse link in $\left(S^{3}, \xi_{s t d}\right)$ is transversely isotopic to a closed braid.

We adopt the usual notation for braid words. The braid group on $b$ strings is generated by $\sigma_{1}, \ldots, \sigma_{b-1}$, so that $\sigma_{i}$ permutes the $i$-th and the $(i+1)$-th strings. We will sometimes write a braid as a braid word, a certain product of the generators $\sigma_{1}, \ldots, \sigma_{b-1}$ and their inverses. The positive resp. negative stabilization of a braid on $b$ strings is formed by adding the $(b+1)$-th string and multiplying the braid word by $\sigma_{b}$ resp. $\sigma_{b}^{-1}$.

Of course, the same link can be represented by different braids. The Markov theorem [Bi] asserts that two braid words describing the same link are related by a sequence of stabilizations, destabilizations and conjugations in the braid group (and, of course, the braid group identities). The Transverse Markov Theorem describes the relation between two braid representations of the same transverse links.

Theorem 1. [Wr, OSh] Let $L_{1}, L_{2}$ be two closed braids which represent transversely isotopic links. Then $L_{2}$ can be obtained from $L_{1}$ by a sequence of positive braid stabilizations and braid isotopies.

The self-linking number $\operatorname{sl}(L)$ is defined as follows. Trivialize the plane field $\xi$, and let the link $L^{\prime}$ be the push-off of $L$ in the direction of the first coordinate vector for $\xi$. Then, $\operatorname{sl}(L)$ is the linking number between $L$ and $L^{\prime}$. Given a closed braid representing $L$, it can be computed as

$$
s l(L)=-b+n_{+}-n_{-},
$$


where $b$ is the braid index, and $n_{+}$and $n_{-}$denote the number of positive resp. negative crossings.

The stabilization of a transverse link can be thought of as negative braid stabilization. Unlike positive braid stabilization, this operation changes the transverse type of the link: if $L^{\prime}$ is the result of stabilization of $L$, then

$$
s l\left(L^{\prime}\right)=\operatorname{sl}(L)-2 .
$$

\section{Khovanov homology}

In this section we give a brief review of Khovanov homology (more or less following the review in [Ra]). Unless otherwise specified, we work with coefficients in $\mathbb{Z}$.

3.1. Khovanov complex. Given a link diagram $L$, we can resolve its crossings so that the result is just the union of planar circles. Each crossing can be resolved in two ways, called the 0 -resolution and the 1-resolution and shown in Fig.1. Let $n$ be the number of crossings of $L$; we will write $n=n_{-}+n_{+}$, where $n_{+}\left(n_{-}\right)$is the number of positive (negative) crossings. (See Fig. 1 for the usual sign conventions.) Then, complete resolutions of $L$ can be conveniently labelled by vertices of the "cube of resolutions" $[0,1]^{n}$. The underlying graded module for the Khovanov complex

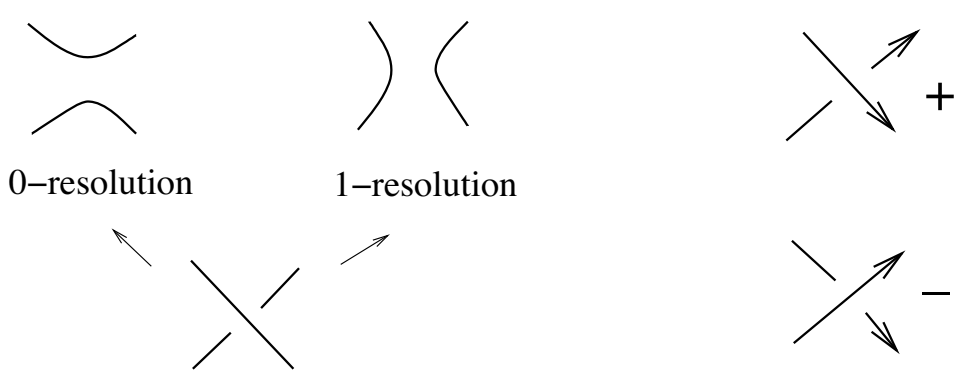

FigURE 1. Resolutions and signs of crossings.

$C K h(L)$ is the direct sum of $\mathbb{Z}$-modules associated to the vertices of $[0,1]^{n}$,

$$
C K h(L)=\oplus_{v \in\{0,1\}^{n}} C K h\left(L_{v}\right) .
$$

Each $C K h\left(L_{v}\right)$ is defined as follows. Let $U$ be the free graded $\mathbb{Z}$-module generated by two elements, $\mathbf{u}_{-}$and $\mathbf{u}_{+}$; the grading $p$ is given by $p\left(\mathbf{u}_{ \pm}\right)= \pm 1$. Suppose that the resolution $L_{v}$ consists of $k$ circles. We then set

$$
C K h\left(L_{v}\right)=U^{\otimes k} .
$$

In other words, $C K h\left(L_{v}\right)$ is freely generated by $k$-tuples obtained by labelling each circle in $L_{v}$ by either $\mathbf{u}_{-}$or $\mathbf{u}_{+}$.

The module $C K h(L)$ is bi-graded. The homological grading, which is constant on each $C K h\left(L_{v}\right)$, is given by $\operatorname{gr}(v)=|v|-n_{-}$, where $|v|$ is the number of 1's in the coordinates of $v$. Besides, there is the quantum grading $q(\mathbf{u})=p(\mathbf{u})+\operatorname{gr}(\mathbf{u})+n_{+}-n_{-}$.

Our next job is to describe the differential $d$ on $C K h(L)$. Loosely, $d$ is the sum of maps $d_{e}$ associated to the edges of $[0,1]^{n}$.

Let $e$ be an edge of $[0,1]^{n}$, and denote by $v_{e}(0)$ resp. $v_{e}(1)$ its initial resp. terminal end. The resolutions $L_{v_{e}(0)}$ and $L_{v_{e}(1)}$ differ in one crossing only (which is 0-resolved 
for $v_{e}(0)$ and 1-resolved for $\left.v_{e}(1)\right)$, and $L_{v_{e}(1)}$ is obtained from $L_{v_{e}(0)}$ in one of two ways: either two circles of $L_{v_{e}(0)}$ merge into one, or one circle splits into two. In the first case, the map $d_{e}: C K h\left(L_{v_{e}(0)}\right) \rightarrow C K h\left(L_{v_{e}(1)}\right)$ is given by multiplication $m: U \otimes U \rightarrow U$, where the two factors of $U \otimes U$ correspond to two circles that merge, and the copy of $U$ in the image corresponds to the resulting circle in $C K h\left(L_{v_{e}(1)}\right)$. In the second case, $d_{e}$ comes from the comultiplication $\Delta: U \rightarrow U \otimes U$, where the $U$ in the domain corresponds to the circle that splits. It remains to define the maps $m$ and $\Delta$ :

$$
\begin{array}{ll}
m\left(\mathbf{u}_{+} \otimes \mathbf{u}_{+}\right)=\mathbf{u}_{+} & \Delta\left(\mathbf{u}_{+}\right)=\mathbf{u}_{+} \otimes \mathbf{u}_{-}+\mathbf{u}_{-} \otimes \mathbf{u}_{+} \\
m\left(\mathbf{u}_{+} \otimes \mathbf{u}_{-}\right)=m\left(\mathbf{u}_{-} \otimes \mathbf{u}_{+}\right)=\mathbf{u}_{-} & \Delta\left(\mathbf{u}_{-}\right)=\mathbf{u}_{-} \otimes \mathbf{u}_{-} . \\
m\left(\mathbf{u}_{-} \otimes \mathbf{u}_{-}\right)=0 &
\end{array}
$$

Now, on the component $C K h\left(L_{v}\right)$ the differential $d$ is defined by

$$
d=\sum_{e: v_{e}(0)=v}(-1)^{s(e)} d_{e}
$$

where the sum is taken over all edges which have $v$ as their initial end. The signs $(-1)^{s(e)}$ are chosen so that $d^{2}=0$ (the choice is not unique, but all the resulting chain complexes are isomorphic).

Khovanov $[\mathrm{Kh}]$ shows that different diagrams for the same knot yield quasiisomorphic chain complexes, so that the isomorphism classes of the (bigraded) homology groups give an invariant of the link. In fact, more is true: as conjectured in $[\mathrm{Kh}]$ and proved in [Ja], Khovanov's theory is functorial, and it follows that there are honest homology groups, not just isomorphism classes, associated to a link. We now turn attention to these the functorial properties.

3.2. Cobordisms and invariance. Given two links and an oriented cobordism between them, there is an induced map between homology groups of the links. We briefly describe this construction.

An oriented cobordism between two links $L^{0}$ and $L^{1}$ is given by an embedded smooth oriented compact surface $S$ in $\mathbb{R}^{3} \times[0,1]$, such that $\partial S=S \cap \partial\left(\mathbb{R}^{3} \times[0,1]\right)$, and $S \cap\left(\mathbb{R}^{3} \times i\right)=L^{i}$ for $i=0,1$. We may assume that $L^{t}=S \cap\left(\mathbb{R}^{3} \times t\right)$ is a link for all but finitely many values of $t$. When $t$ passes through the critical value, the isotopy type of the link changes by a Morse move, and the surface $S^{t}=\left(S \cap \mathbb{R}^{3} \times[0, t]\right)$ changes by an attachment of a handle (of index 0,1 , or 2 ). Further, we can fix a projection $\mathbb{R}^{3} \rightarrow \mathbb{R}^{2}$, and assume that it gives a regular projection for $L^{t}$ for all but finitely many special values of $t$ (and that the set of these special values is disjoint from the set of the Morse critical values). Thus, we obtain link diagrams (still denoted $\left.L^{t}\right)$. When $t$ passes through a special value where the projection of the link is not regular, the link remains the same, but its diagram changes by a Reidemeister move. The isomorphism class of the surface $S^{t}$ remains unchanged.

Therefore, the cobordism $S$ can be represented as a sequence of elementary cobordisms,

$$
S=S_{1} \cup S_{2} \cup \cdots \cup S_{k},
$$

where each cobordism $S_{i}$ between two diagrams $L^{t_{i}}$ and $L^{t_{i+1}}$ corresponds to either a Reidemester move or a handle attachment. Now, each $S_{i}$ induces a map 
$f_{S_{i}}: K h\left(L^{t_{i}}\right) \rightarrow K h\left(L^{t_{i+1}}\right)$. For Reidemeister moves, $f_{S_{i}}$ comes from the quasiisomorphisms between chain complexes $C K h\left(L^{t_{i}}\right)$ and $C K h\left(L^{t_{i+1}}\right)$ mentioned in the previous section (we describe these quasi-isomorphisms in a little more detail in section 4). For Morse moves, $f_{S_{i}}$ is defined as follows $[\mathrm{Kh}]$. We need two additional maps, $\iota: \mathbb{Z} \rightarrow U$ and $\epsilon: U \rightarrow \mathbb{Z}$, defined by

$$
\begin{array}{ll}
\epsilon\left(\mathbf{u}_{-}\right)=1 & \iota(1)=\mathbf{u}_{+} \\
\epsilon\left(\mathbf{u}_{+}\right)=0 &
\end{array}
$$

Now, the attachment of a 0-handle corresponds to a "birth" of a circle in the diagram, and the map on the chain complex is given by $\iota$ (for all possible resolutions). Similarly, the attachment of a 2-handle (the "death" of a circle) gives the map given by $\epsilon$. The attachment of 1-handle is given by $m$ or $\Delta$ on each component of the chain complex, depending on whether the 1-handle merges two circles of a particular resolution or splits one circle into two. (Note that the differential in Khovanov's theory is defined in a similar way: two resolutions given by adjacent vertices of $[0,1]^{n}$ differ precisely by the attachment of a 1-handle.) Finally, the map $f_{S}$ is defined as the composition of the maps induced by the elementary cobordisms,

$$
f_{S}=f_{S_{k}} \circ \cdots \circ f_{S_{2}} \circ f_{S_{1}} .
$$

Jacobsson $[\mathrm{Ja}]$ proves that up to a sign, the map $f_{S}$ depends on the isotopy class of $S$ rel $\partial S$ only, that is, if

$$
S=S_{1} \cup S_{2} \cup \cdots \cup S_{k} \text { and } S=S_{1}^{\prime} \cup S_{2}^{\prime} \cup \cdots \cup S_{k^{\prime}}^{\prime}
$$

are two decompositions of $S$ into elementary cobordisms, then

$$
f_{S_{k}} \circ \cdots \circ f_{S_{2}} \circ f_{S_{1}}= \pm f_{S_{k^{\prime}}^{\prime}} \circ \cdots \circ f_{S_{2}^{\prime}} \circ f_{S_{1}^{\prime}} .
$$

In particular, if two diagrams of a link are related by a sequence of Reidemeister moves, then the induced isomorphism between the homology groups is canonical up to a sign.

\section{Definition of the invariant}

In this section we define the transverse link invariant $\psi(L) \in K h(L)$.

First, we fix a braid diagram $L$ for our link, and pick a distinguished element $\tilde{\psi}(L)$ in the chain complex $C K h(L)$. We will check that $\tilde{\psi}(L)$ is a cycle, so that it defines an element $\psi(L)$ of the homology group $K h(L)$. Finally, we show that $\psi(L)$ does not depend on the choice of the braid diagram and remains the same under transverse link isotopies. This means that $\psi(L)$ is indeed an invariant of the transverse link.

Given a braid diagram $L$ for our link, we choose a resolution which is given by $b$ parallel strings: that is, we take the 0-resolution for each positive crossing and the 1-resolution for each negative crossing of $L$. Note that this is the oriented resolution of the diagram; we denote it by $L_{o}$.

We set

$$
\tilde{\psi}(L)=\mathbf{u}_{-} \otimes \mathbf{u}_{-} \otimes \cdots \otimes \mathbf{u}_{-} \in U^{\otimes b}=C K h\left(L_{o}\right) .
$$

Proposition 1. The element $\tilde{\psi}(L)$ is a cycle in $(C K h(L), d)$. 
Proof. The differential $d$ on $C K h\left(L_{v}\right)$ is the sum of maps for all edges $e$ which have $v$ as their initial end. By our choice of the resolution $L_{v}$, such edges correspond to positive crossings. Moreover, when a 0-resolution of a positive crossing is changed into a 1-resolution, the two circles of $L_{v}$ which are "connected" by this crossing merge into one. This means that each map $d_{e}$ is given by multiplication, and then

$$
d_{e}(\tilde{\psi}(L))=m\left(\mathbf{u}_{-} \otimes \mathbf{u}_{-}\right)=0 .
$$

Taking the sum over all positive crossings, we see that $d(\tilde{\psi}(L))=0$.

Proposition 2. $\psi(L) \in K h^{0, s l(L)}$.

Proof. By construction, $\psi(L)$ is a homogeneous element. The homological and quantum gradings are easy to compute: since the number of 1's for the chosen resolution is exactly the number of negative crossings, $\operatorname{gr}(\psi(L))=0$. Now, $p(\psi(L))$ is the braid index of $L$, and the formula $q(\psi(L))=s l(L)$ is an immediate consequence of (1) and the definition of the quantum grading.

Now we want to check that $\psi(L)$ is independent of a particular braid representation of the transverse knot. The Transverse Markov Theorem says that the braids representing two transversely isotopic knots are related by a sequence of positive stabilizations and braid isotopies. The two braid words will then be related via positive stabilizations, conjugations and the braid group identities. For the corresponding braid diagrams, this yields a sequence of "transverse Reidemeister moves", as follows. Positive stabilization gives the move (R1) with a positive crossing introduced (the other version of (R1) is not allowed). The braid isotopies give the (R2) and (R3) moves, all versions of which are allowed. In the following Lemma, we check that the moves (R1)-(R3) respect $\psi(L)$ by analyzing the effect of each move on Khovanov's homology. If we were dealing with knots, it would suffice to consider only the versions of (R1)-(R3) shown in Fig. 2, since all the other versions can be obtained by a combination of these three. With braids, more care is needed: there is another version of (R2) obtained by turning our picture upside down; since we cannot turn braids upside down, we actually need to consider both versions of (R2). However, the two proofs are identical, so we only give one of them. Also, it is not hard to check that all possible versions of the (R3) move can be reduced to the one shown by a combination of (R2) moves.

Lemma 1. Let $L$ and $L^{\prime}$ be two braid diagrams related by one of the three transverse Reidemeister moves (R1), (R2), (R3), and denote by $\rho_{i}: C K h(L) \rightarrow C K h\left(L^{\prime}\right)$, $i=1,2,3$, the associated quasi-isomorphisms between the two chain complexes. Then

$$
\rho_{i}(\tilde{\psi}(L))= \pm \tilde{\psi}\left(L^{\prime}\right)
$$

Proof. We recall how the quasi-isomorphisms $\rho_{i}$ are constructed in [Kh], and see what happens to the distinguished element $\tilde{\psi}(L)$.

(R1) move: The complex $C K h\left(L^{\prime}\right)$ decomposes as a direct sum $X_{1} \oplus X_{2}$, where the $X_{2}$ is acyclic, and $X_{1}$ is isomorphic to $C K h(L)$ (Fig. 3). The isomorphism $\rho_{1}$ is given by

$$
\begin{aligned}
& \rho_{1}\left(\mathbf{u}_{-}\right)=\mathbf{u}_{-} \otimes \mathbf{u}_{-} \\
& \rho_{1}\left(\mathbf{u}_{+}\right)=\mathbf{u}_{+} \otimes \mathbf{u}_{-}-\mathbf{u}_{-} \otimes \mathbf{u}_{+},
\end{aligned}
$$

and we see that $\tilde{\psi}(L)$ is mapped to $\tilde{\psi}\left(L^{\prime}\right)$. 
$\mathrm{R} 1$

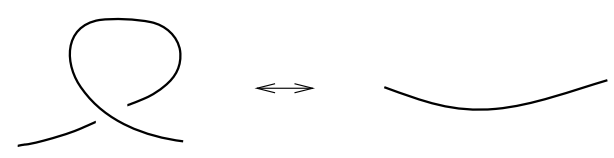

R3

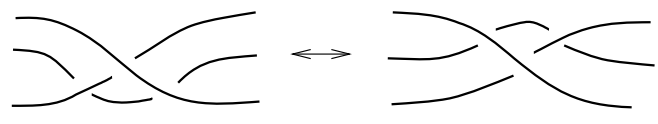

$\mathrm{R} 2$

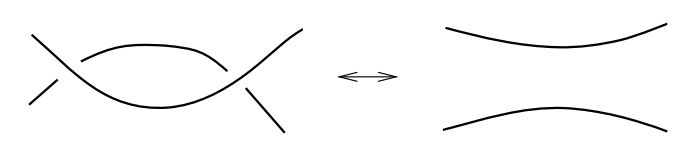

Figure 2. Reidemeister moves in the transverse braid setting.
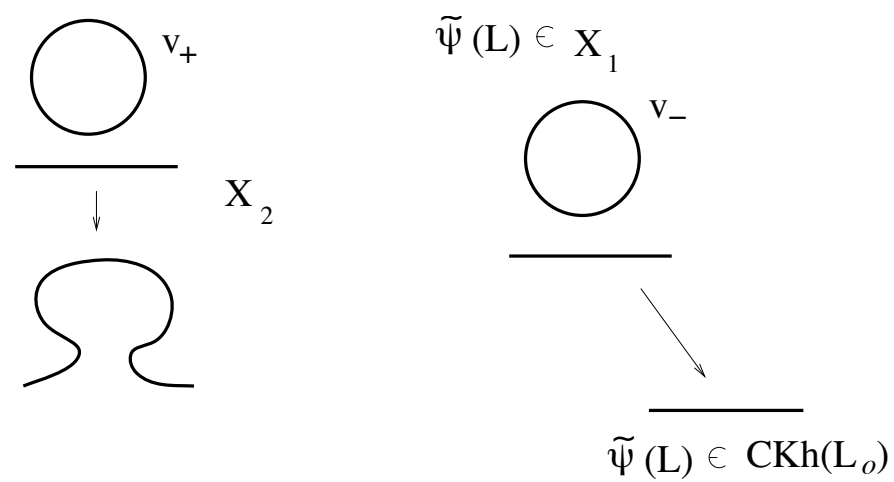

Figure 3. Construction of the quasi-isomorphism $\rho_{1}$.

(R2) move: The four possible resolutions of the two extra crossings of $L^{\prime}$ are shown in Fig. 4. Again, the complex $C K h\left(L^{\prime}\right)$ decomposes as $X_{1} \oplus X_{2} \oplus X_{3}$, where $X_{2}$ and $X_{3}$ are both acyclic, and $X_{1}$ isomorphic to $C K h(L)$ via the isomorphism $\rho_{2}: C K h(L) \rightarrow C K h\left(L^{\prime}\right)$ given by

$$
\rho_{2}(x)=(-1)^{\operatorname{gr}(x)}\left(x+\iota\left(d_{e}(x)\right)\right) .
$$

(The map $\iota$ defined in section 3.2 and the map $\partial_{e}$ corresponding to the edge $e$ are shown in the Figure, and the oriented resolution of $L$ is naturally identified with the oriented resolution of $L^{\prime}$, so that $x$ on the right-hand side actually lives in $\left.C K h\left(L^{\prime}\right)\right)$.

We see that $\rho_{2}$ maps $\tilde{\psi}(L)$ to $\pm\left(\tilde{\psi}\left(L^{\prime}\right)+\iota\left(d_{e}\left(\tilde{\psi}\left(L^{\prime}\right)\right)\right)\right.$. In the proof of Proposition 1 , we've checked that $d_{e}\left(\tilde{\psi}\left(L^{\prime}\right)\right)=0$. It follows that up to a sign, $\tilde{\psi}(L)$ is mapped to $\tilde{\psi}\left(L^{\prime}\right)$.

(R3) move: Now we have decompositions $C K h(L)=X_{1} \oplus X_{2} \oplus X_{3}$ and $C K h\left(L^{\prime}\right)=$ $X_{1}^{\prime} \oplus X_{2}^{\prime} \oplus X_{3}^{\prime}$ where $X_{2}, X_{3}, X_{2}^{\prime}, X_{3}^{\prime}$ are all acyclic, and there is an isomorphism $\rho_{3}: X_{1} \rightarrow X_{1}^{\prime}$.

We briefly describe how $X_{1}$ is formed. First, pick 1-resolutions of crossings $\mathrm{r}$ and $r^{\prime}$ (note that the resulting diagrams are isomorphic). With this fixed, consider all 


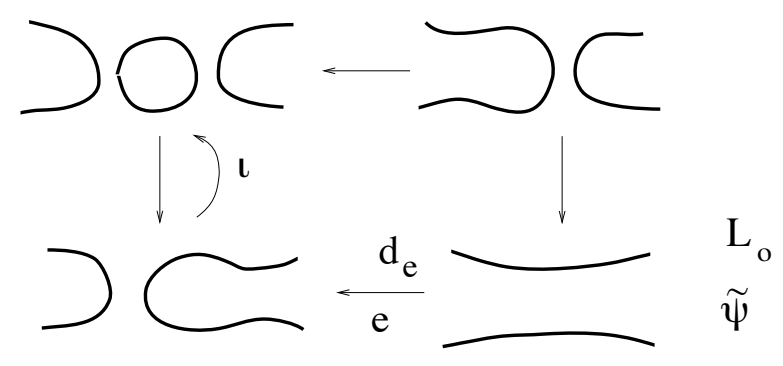

FIGURE 4. Construction of the quasi-isomorphism $\rho_{2}$.

possible resolutions of other crossings, and denote the direct sum of the associated components of $C K h(L)$ resp. $C K h\left(L^{\prime}\right)$ by $C K h\left(L_{* 1}\right)$ resp. $C K h\left(L_{* 1}^{\prime}\right)$. (This is not a subcomplex.) Next, denote by $C K h\left(L_{* 100}\right)$ the part of $C K h(L)$ arising from all
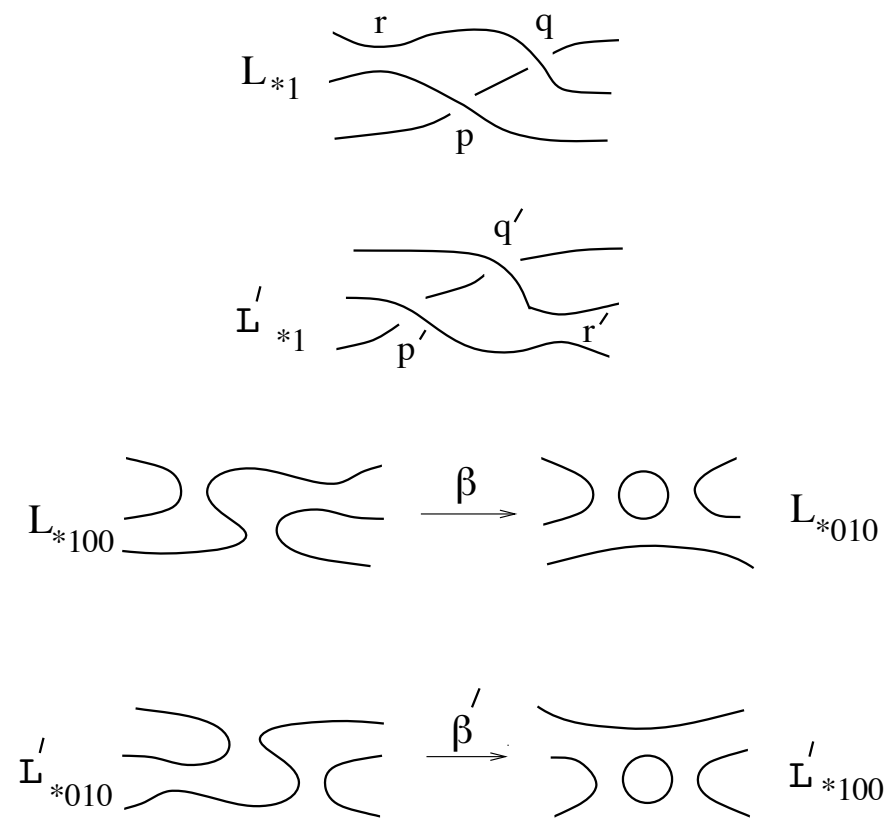

Figure 5. Construction of complexes $X_{1}$ and $X_{1}^{\prime}$.

complete resolutions of $L$ with a 1-resolution at $\mathrm{p}$ and 0-resolutions at both $\mathrm{q}$ and $\mathrm{r}$; form $C K h\left(L_{* 010}\right), C K h\left(L_{* 100}^{\prime}\right)$ and $C K h\left(L_{* 010}^{\prime}\right)$ by analogy. Now, define

$$
\begin{aligned}
& X_{1}=\left\{x+\beta(x)+y \mid x \in C K h\left(L_{* 100}\right), y \in C K h\left(L_{* 1}\right)\right\} \\
& X_{1}^{\prime}=\left\{x+\beta(x)+y \mid x \in C K h\left(L_{* 010}^{\prime}\right), y \in C K h\left(L_{* 1}^{\prime}\right)\right\},
\end{aligned}
$$

where $\beta: C K h\left(L_{* 100}\right) \rightarrow C K h\left(L_{* 010}\right)$ and $\beta: C K h\left(L_{* 010}^{\prime}\right) \rightarrow C K h\left(L_{* 100}^{\prime}\right)$ are certain chain maps. The isomorphism $\rho_{3}: X_{1} \rightarrow X_{1}^{\prime}$ is given by

$$
\rho_{3}(x+\beta(x)+y)=x+\beta^{\prime}(x)+y,
$$


where the natural identifications between $C K h\left(L_{* 100}\right)$ and $C K h\left(L_{* 010}^{\prime}\right)$ etc. are used. We do not describe the maps $\beta$ and $\beta^{\prime}$, referring the reader to [Kh]: the only thing we need to know is that $\tilde{\psi}(L) \in C K h\left(L_{* 1}\right)$ and $\tilde{\psi}\left(L^{\prime}\right) \in C K h\left(L_{* 1}^{\prime}\right)$, so $\rho_{3}(\tilde{\psi}(L))=\tilde{\psi}\left(L^{\prime}\right)$.

We've checked that the distinguished element $\psi(L) \in K h(L)$ behaves nicely under the three transverse Reidemeister moves, and we know that any two transversely isotopic knots are related by a sequence of such moves, but why would an arbitrary transverse isotopy between $L$ and $L^{\prime}$ send $\psi(L)$ to $\psi\left(L^{\prime}\right)$ ? We have to give the Transverse Markov Theorem another look: in $[\mathrm{Wr}]$ it is actually shown that an arbitrary transverse isotopy $S$ can be smoothly modified into a composition of braid isotopies and positive stabilizations while the two links are fixed. Then, up to an isotopy of $S$ rel $\partial S$, the cobordism $S$ between $L$ and $L^{\prime}$ decomposes as $S_{1} \cup \cdots \cup S_{k}$, and Jacobsson's theorem from section 3.2 implies that

$$
f_{S}(\psi(L))=f_{S_{k}} \circ \cdots \circ f_{S_{2}} \circ f_{S_{1}}(\psi(L))= \pm \psi\left(L^{\prime}\right) .
$$

We have proved the following

Theorem 2. The element $\psi(L) \in K h(L)$ is an invariant of the transverse link $L \in$ $\left(S^{3}, \xi_{\text {std }}\right)$, well-defined up to a sign.

\section{Properties of $\psi(L)$}

\subsection{Transverse stabilization.}

Theorem 3. If $L$ is the transverse stabilization of another transverse link, then $\psi(L)=0$.
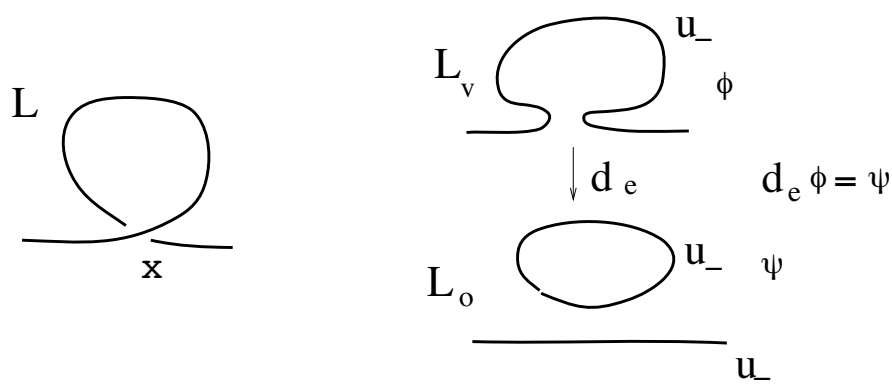

Figure 6. Transverse stabilization and Khovanov's complex.

Proof. We construct an element $\tilde{\phi} \in C K h(L)$ such that $d \tilde{\phi}=\tilde{\psi}(L)$. Since $L$ is the result of a transverse stabilization (that is, an addition to the braid of an extra string and an extra negative crossing $\mathrm{x}$ ), it has a diagram with a "negative kink" as shown on Fig. 6. For the oriented resolution $L_{o}$, we take the 1-resolution of the crossing $\mathrm{x}$. Let $e$ be the edge of the cube of resolutions corresponding to $\mathrm{x}$; then $o$ is the terminal end of $e$. We denote by $v$ the initial end of $e$. In other words, we take the 0-resolution 
of $\mathrm{x}$ to form $L_{v}$, and all the other crossings are resolved as in $L_{o}$. Now, label all the components of $L_{v}$ by $\mathbf{u}_{-}$, and set

$$
\tilde{\phi}=\mathbf{u}_{-} \otimes \ldots \mathbf{u}_{-} \in C K h\left(L_{v}\right) .
$$

We compute $d \tilde{\phi}$ as follows. The component $d_{e}: C K h\left(L_{v}\right) \rightarrow C K h\left(L_{o}\right)$ of $d$ is given by comultiplication $\Delta$, since the change of the resolution for $\mathrm{x}$ splits a circle into two. Thus, $d_{e} \tilde{\phi}=\tilde{\psi}$. Furthermore, similar to proof of Proposition 1, all the other terms of $d$ on the component $C K h\left(L_{v}\right)$ correspond to positive crossings and are given by multiplication maps, which send $\tilde{\phi}$ to 0 . It follows that $d \tilde{\phi}=\tilde{\psi}$, as required.

\subsection{Positive crossing resolution.}

Theorem 4. Suppose that the transverse braid $L^{2}$ is obtained from the transverse braid $L^{1}$ by resolving a positive crossing (note that it has to be a 0-resolution). Let $S$ be the resolution cobordism, and $f_{S}: K h\left(L^{1}\right) \rightarrow K h\left(L^{2}\right)$ the associated map on homology. Then

$$
f_{S}\left(\psi\left(L^{1}\right)\right)= \pm \psi\left(L^{2}\right) .
$$

Proof. The cobordism $S$ is a composition of a 1-handle attachment and a Reidemeister move (R1), as shown on Fig. 7. On the component $C K h\left(L_{o}^{1}\right)$ of the Khovanov's complex for $L^{1}$, the 1-handle attachment induces a map given by comultiplication $\Delta$, since the handle splits a circle on the oriented resolution. The map induced by the (R1) move was analyzed in the proof of Lemma 1. It follows that the element $\tilde{\psi}\left(L^{1}\right)$, given by the $\mathbf{u}_{-}$labels of all circles for $L_{o}^{1}$, is mapped to $\tilde{\psi}\left(L^{2}\right)$ (given by the $\mathbf{u}_{-}$ labels on circles for $L_{o}^{2}$ ).

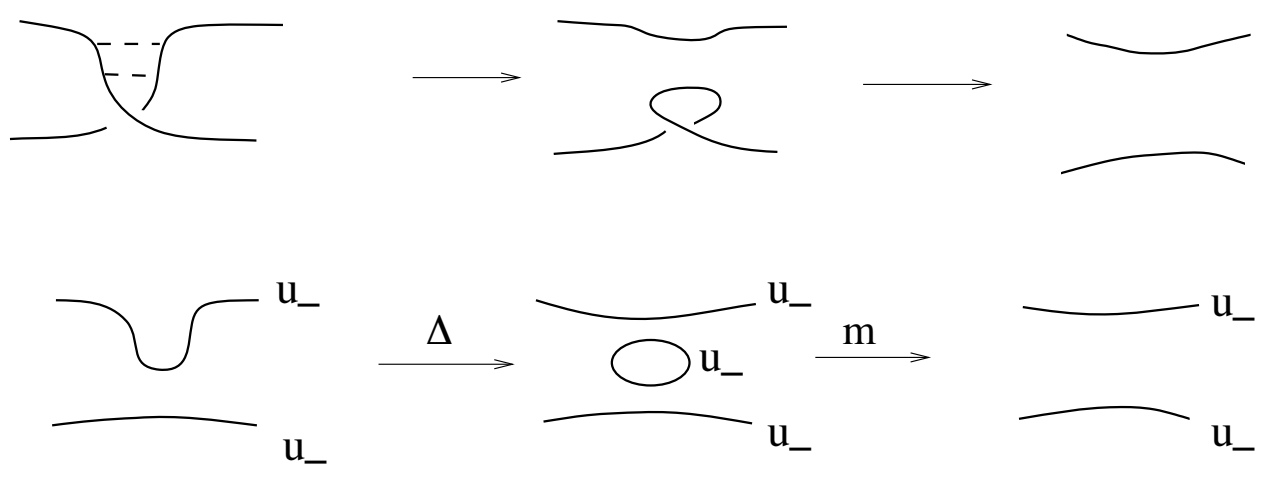

Figure 7. Resolving a positive crossing.

Recall that a braid is called quasipositive [Ru] if its braid word is a product of conjugates of the form $w \sigma_{i} w^{-1}$, where $w$ is an arbitrary element of the braid group.

Corollary 1. If $L$ is represented by a quasipositive braid, then $\psi(L) \neq 0$. Moreover, it is a primitive non-torsion element of $K h(L)$. 
Proof. Resolving a few positive crossings, we convert the braid representing $L$ into a braid equivalent to a trivial one (of the same braid index). For the trivial braid $\mathcal{O}$, there are no differentials in the chain complex, and $\psi(\mathcal{O})$ is a generator of $K h^{0, s l(\mathcal{O})}=$ $\mathbb{Z}$. Since $\psi(L)$ is mapped to $\psi(\mathcal{O})$, it must be non-torsion and primitive.

Remark 1. Let $L$ be a positive braid of braid index $b$ with $n$ crossings. Then, the homology of $L$ lies in non-negative homological degrees, with $K h^{0, n-b}=\mathbb{Z}$, $K h^{0, n-b+2}=\mathbb{Z}$ and no other homology in $K h^{0, *}$. The element $\psi(L)$ is a generator of $K h^{0, n-b}$.

Corollary 2. A transverse link $L$ represented by a quasipositive braid cannot be obtained by a transverse stabilization of any other link.

Remark 2. Corollary 2 follows easily from the fact that a quasipositive braid maximizes the self-linking number in its transverse link type. More precisely, for an arbitrary transverse link $L$ the slice-Bennequin inequality [Ru] gives

$$
\operatorname{sl}(L) \leq-\chi(\Sigma)
$$

where $\Sigma \subset B^{4}$ is a surface with boundary $\partial \Sigma=L \in S^{3}=\partial B^{4}$; for quasipositive braids (7) becomes an equality. This bound was first proved by Rudolph by means of gauge theory. It is interesting to note that it can be obtained purely by Khovanovhomological methods. Indeed, as in [Ru], it is straightforward to reduce the question to the case of a positive braid representing a torus knot $T_{p, q}$ (introducing positive crossings and keeping track of how both sides of (7) change). Then, the self-linking number is easily seen to be $2 g\left(T_{p, q}\right)-1$ (where $g$ denotes genus), and $g_{*}\left(T_{p, q}\right)=$ $g\left(T_{p, q}\right)$. The last identity is the Milnor conjecture, whose Khovanov homology proof was recently obtained by Rasmussen [Ra] (the original gauge-theoretic proof is due to Kronheimer and Mrowka [KM]).

5.3. Negative crossings. The following Proposition is useful for calculations and shows that the invariant $\psi$ vanishes for many transverse links.

Proposition 3. Suppose that the transverse link $L$ is represented by a closed braid whose braid word contains a factor of $\sigma_{i}^{-1}$ but no $\sigma_{i}$ 's for some $i=1, \ldots, n$. (This means that all the crossings in the braid diagram on the level between $(i-1)$-th and $i$-th string are negative.) Then $\psi(L)=0$.

Proof. First of all, we delete all $\sigma_{i}^{-1}$ but one from the braid word, obtaining a link that decomposes as a connected sum of two links (connected by a negative crossing, the $\sigma_{i}^{-1}$ that remains). Then, we delete negative crossings from and insert positive crossings into both components of the connected sum, obtaining as a result two positive torus knots connected by a negative crossing. This is illustrated on Fig. 8. Denote the obtained transverse link by $L^{\prime}$. By Theorem 4, it suffices to show that $\psi\left(L^{\prime}\right)=0$. Topologically, the link $L^{\prime}$ is just the connected sum of two torus knots, but its selflinking number is not maximal (because we can connect the two components by a positive crossing to increase $s l$ ). Connected sums of torus knots are transversely simple [EH2, Et], so $L^{\prime}$ is the transverse stabilization of another link. By Theorem 3, $\psi\left(L^{\prime}\right)=0$. 
$\mathrm{L}$
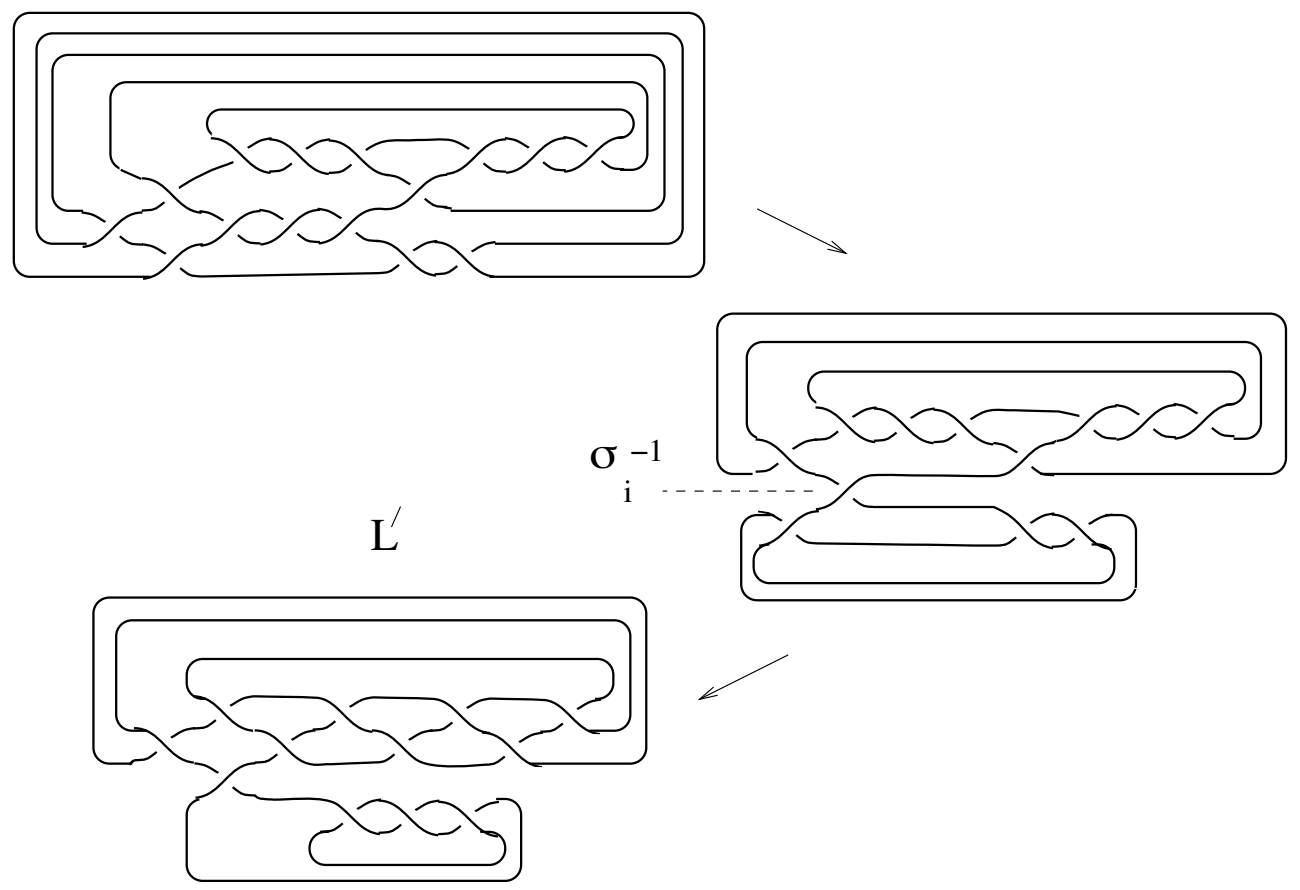

FiguRe 8. From $L$ to $L^{\prime}$.

\section{Examples}

Example 1. For $q>0,|p| \geq q$ let $L$ be a transverse link of the $(p, q)$-torus link type. (1) Suppose $p>0$. If $L$ maximizes the self-linking number in its smooth link type, i.e. $\operatorname{sl}(L)=p q-p-q$, then $\psi(L)$ is a generator of $K h^{0, p q-p-q}=\mathbb{Z}$. Otherwise $\psi(L)=0$. (2) If $p<0$, then $\psi(L)$ vanishes.

Proof. (1) We use transverse simplicity of positive torus links [EH1, Et]. The (unique) transverse positive $(p, q)$-torus link with $s l(L)=p q-p-q$ is represented by a positive braid with $q$ strings and $p(q-1)$ crossings, so the result follows from Remark 1 . If $s l(L)<p q-p-q$, then $L$ is obtained by transverse stabilization, so $\psi(L)=0$ by Theorem 3.

(2) Follows from Proposition 3.

Example 2. Consider the transverse braids $L^{1}=\sigma_{1}^{2 p+1} \sigma_{2}^{2 q} \sigma_{1}^{2 r} \sigma_{2}^{-1}$ and $L^{2}=$ $\sigma_{1}^{2 p+1} \sigma_{2}^{-1} \sigma_{1}^{2 r} \sigma_{2}^{2 q}$. It is shown in $[\mathrm{BM}]$ that $L^{1}$ and $L^{2}$ are not transversely isotopic when $p, q, r>1, p+1 \neq q \neq r$ (although they are smoothly isotopic, and $\left.\operatorname{sl}\left(L^{1}\right)=\operatorname{sl}\left(L^{2}\right)\right)$. However, we have $\psi\left(L^{1}\right)= \pm \psi\left(L^{2}\right)$. Indeed, both $\psi\left(L^{1}\right)$ and $\psi\left(L^{2}\right)$ are generators of $K h^{0, s l\left(L^{i}\right)}=\mathbb{Z}$.

Proof. Because of Proposition 2 and Corollary 1, we only need to check that $K h^{0, s l}=$ $\mathbb{Z}$. In fact, $K h^{0, s l}(L)$ has rank one and $K h^{-1, *}(L)=0$ for any link diagram $L$ with only one negative crossing (we assume that this crossing corresponds to some $\sigma_{i}^{-1}$, 
and there is another appearance of $\sigma_{i}$ in the braid word for $L$ ). This can be shown by a direct analysis of the relevant part of $C K h(L)$. We sketch the proof for the case of coefficients in $\mathbb{Z} / 2 \mathbb{Z}$; for coefficients in $\mathbb{Z}$, an appropriate choice of signs needs to be fixed.

Let $L$ stand for $L^{1}$ or $L^{2}$. For the given diagram $C K h^{-1, s l}(L)$ has rank one and is generated by $\mathbf{u}_{-} \otimes \mathbf{u}_{-}$in the complete resolution of $L$ given by 0 -resolution of all crossings (this resolution consists of two circles and is shown on Fig. 9). Similarly,

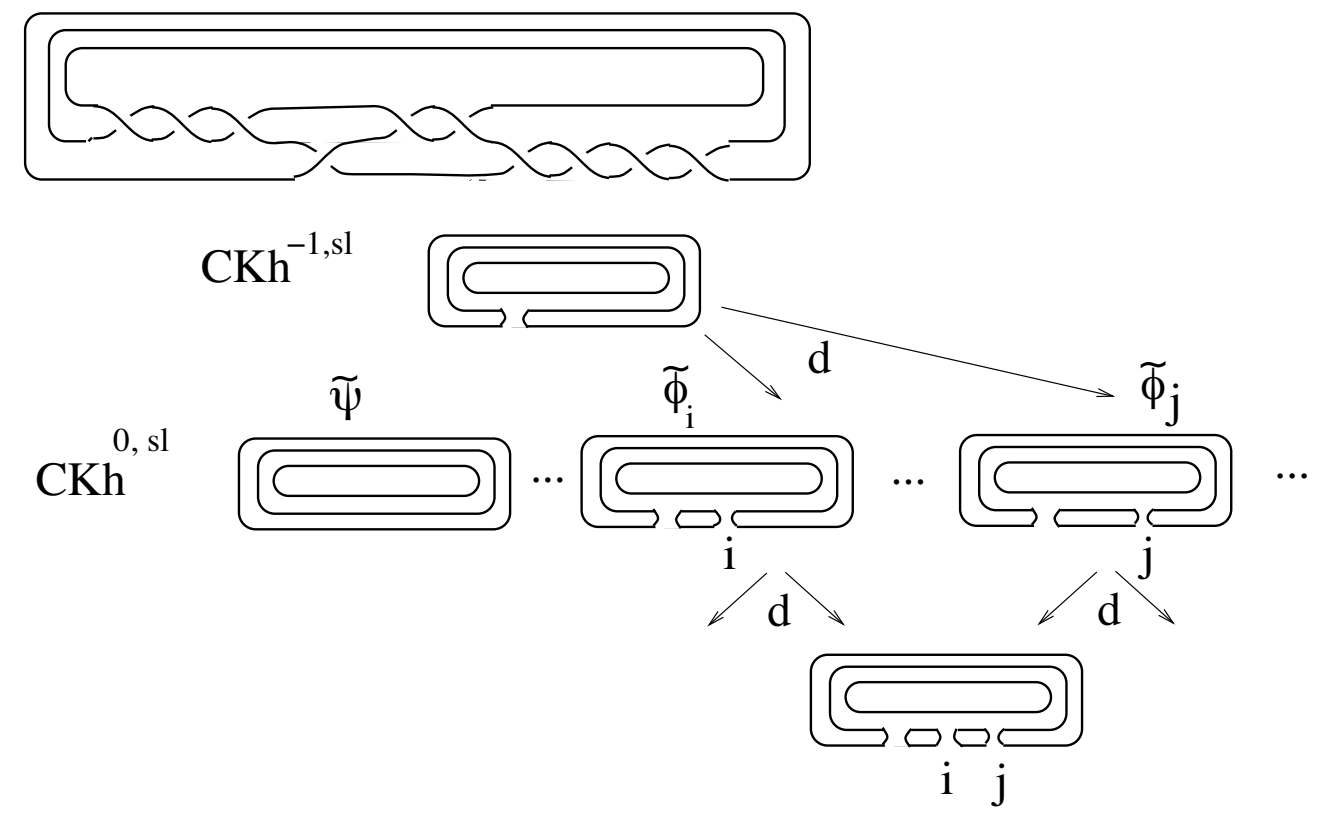

Figure 9. Computing $K h^{0, s l}(L)$. Each component of complete resolutions shown must be labelled by $\mathbf{u}_{-}$.

$C K h^{0, s l}(L)$ has rank $2 q+1$, with generators given by $\tilde{\psi}(L)$ and $2 q$ elements $\tilde{\phi}_{i}$. The elements $\tilde{\phi}_{i}$ are formed by the $\mathbf{u}_{-}$labels on the complete resolutions obtained as follows: the crossing that corresponds to the $i$-th $\sigma_{2}$ in the product $\sigma_{2}^{2 q}$ is 1-resolved, all other crossings are 0-resolved. Further, $d\left(\tilde{\phi}_{i}\right)$ in turn comes from 1-resolutions of the $j$-th crossing in $\sigma_{2}^{2 q}$ for all $j \neq i$. It follows that, apart from $\tilde{\psi}(L)$, the only cycle in $C K h^{0, s l}(L)$ is $\phi_{1}+\phi_{2}+\cdots+\phi_{2 q}$. This cycle is homologous to $\tilde{\psi}(L)$, since the boundary of the generator of $C K h^{-1, s l}(L)$ is equal to $\tilde{\psi}(L)+\phi_{1}+\phi_{2}+\cdots+\phi_{2 q}$.

\section{A bound on the self-linking number}

In this section we obtain a bound on the self-linking number of a transverse knot $K$ in terms of the knot invariant $s(K)$ introduced by Rasmussen [Ra]. As we mention below, $s(K)$ is defined as a certain quantum grading in Lee's version of the Khovanov homology [Lee]. (In Lee's construction, which works for rational coefficients only, the generators for the complex $C K h^{\prime}(K)$ and the gradings are the same as in $C K h(K)$, but the differential is different.) Rasmussen conjectured that this invariant coincides 
with the $s(K)$ invariant of Bar-Natan [BN], and is twice the $\tau(K)$ invariant of Ozsváth and Szabó [OS1]. (However, after the present paper was written, Rasmussen's conjecture was disproved in $[\mathrm{HO}]$.) Most importantly, $|s(K)|$ gives a lower bound for the slice genus. If $K$ is alternating, $s(K)$ is simply the signature of the knot.

Proposition 4. For any transverse knot $K$

$$
s l(K) \leq s(K)-1 .
$$

Proof. As Jake Rasmussen pointed out to the author, the invariant $s(K)$ in [Ra] is defined as $s(K)=\max q(\tilde{x})+1$, where $\tilde{x}$ is an element of $C K h^{\prime}(K)$ homologous (in $\left.C K h^{\prime}(K)\right)$ to an element of the form $\tilde{\psi}(K)+y$, where $q(y)>q(\psi(\tilde{\psi}))$. (Note that Lee's differential does not preserve the quantum grading.) Since $q(\tilde{\psi})=\operatorname{sl}(K)$ for a transverse knot $K$, it follows immediately that $s l(K) \leq s(K)-1$.

Remark 3. Proposition 4 gives an improvement for the well-known Thurston-Bennequin [Be] and slice-Bennequin [Ru] bounds on $\operatorname{sl}(K)$. The relation between Rasmussen's invariant and the slice-Bennequin inequality was independently established by A. Shumakovich [Sh].

Corollary 3. If $K$ is alternating, $\operatorname{sl}(K) \leq \sigma(K)-1$, where $\sigma(K)$ is the signature of the knot.

Remark 4. Since every bound for the self-linking number of transverse knots is automatically a bound for $t b(K)+|r(K)|$, the Thurston-Bennequin and rotation numbers of Legendrian knots (Legendrian and transverse knots are related by pushoffs [Et]), for an alternating Legendrian knot $K$ we have

$$
t b(K)+|r(K)| \leq \sigma-1 .
$$

This bound was obtained in [Pl1] via Heegaard Floer homology techniques. Indeed, it is a special case of the inequality

$$
t b(K)+|r(K)| \leq 2 \tau(K)-1,
$$

where $\tau(K)$ is the Ozsváth-Szabó invariant [OS1]. The latter bound, together with Proposition 4, emphasizes the similarity between $s(K)$ and $2 \tau$, and gives yet another connection between the Heegaard Floer theory and the Khovanov homology.

\section{Reduced homology and a relation to Ozsváth-Szabó invariants}

A transverse link invariant can also be defined in the reduced version of the Khovanov homology. We recall the construction of the reduced complex. Starting with a link $L$ with a marked point on it, consider the usual Khovanov complex $C K h(L)$. For each complete resolution of $L$, exactly one of the circles contains the marked point. Let $C K h_{\mathbf{u}_{-}}(L)$ be the subcomplex generated by those generators of $C K h(L)$ that have the label $\mathbf{u}_{-}$on the marked circle. Then, the reduced chain complex is the factor $\widetilde{C K h}(L)=C K h(L) / C K h_{\mathbf{u}_{-}}(L)$, and $\widetilde{K h}(L)$ is the corresponding homology. When the homology is taken with coefficients in $\mathbb{Z} / 2 \mathbb{Z}, \widetilde{K h}(L)$ is independent of the choice of the marked point.

To define the transverse link invariant $\psi(L)$ in the reduced homology, we observe that $\widetilde{C K h}(L) \cong C K h_{\mathbf{u}_{-}}(L)$; then, we can again define $\psi(L)$ as the class of the element obtained by labeling with $\mathbf{u}_{-}$each component of the oriented resolution. 
The "reduced" invariant $\psi(L) \in \widetilde{K h}(L)$ has the same properties as those we proved in the non-reduced case (the proofs would be identical).

In the Introduction, we mentioned the connection between $\widetilde{K h}(L)$ and the Heegaard Floer homology of the 3-manifold $\Sigma(L)$, which is the double cover of $S^{3}$ branched over a smooth link $L$. As shown by Oszváth and Szabó [OS2], there exist a spectral sequence whose $E^{2}$ term is $\widetilde{K h}(L)$, and $E^{\infty}$ term is the Heegaard Floer homology $\widehat{H F}(-\Sigma(L))$ (the coefficients for both homology theories are taken in $\mathbb{Z} / 2 \mathbb{Z}$ ). More precisely, for a fixed link diagram, the construction of [OS2] gives a filtered chain complex $C(L)$, whose homology is $\widehat{H F}(-\Sigma(L))$, and the associated graded complex is the chain complex $\widetilde{C K h}(L)$ for reduced Khovanov homology (with its homological grading). When $L$ is alternating, the spectral sequence collapses, yielding a canonical isomorphism between $\widetilde{K h}(L)$ and the associated graded group of $\widehat{H F}(-\Sigma(L))$. (For coefficients in a field, the associated graded group of $\widehat{H F}(-\Sigma(L))$ is of course isomorphic to $\widehat{H F}(-\Sigma(L))$, although non-canonically).

When $S^{3}$ is equipped with the standard contact structure and $L$ is a transverse link, the manifold $\Sigma(L)$ carries a natural contact structure $\xi_{L}$ lifted from $S^{3}$. In a related paper [Pl2], we study the Ozsváth-Szábo contact invariant $c\left(\xi_{L}\right) \in \widehat{H F}(-\Sigma(L))$, associated to the contact structure. It turns out that the properties of $c\left(\xi_{L}\right)$ are very similar to those of $\psi(L)$; in particular, the results of section 5 hold true for $c\left(\xi_{L}\right)$. It is natural to conjecture that the element $c\left(\xi_{L}\right)$ "corresponds" to $\psi(L)$ under the spectral sequence. To be more precise, we consider the case of alternating knots. We have seen that $\psi(L)$ is a homogeneous element of $\widetilde{K h}(L)$ of homological degree 0 . We hope that the filtration level $c\left(\xi_{L}\right)$ in $\widehat{H F}(-\Sigma(L))$ matches the homological degree of $\psi(L)$; denoting by $c_{0}\left(\xi_{L}\right)$ the image of $c\left(\xi_{L}\right)$ in the component $\widehat{H F}_{0}(-\Sigma(L)) / \widehat{H F}_{1}(-\Sigma(L))$ of the associated graded group, we suggest

Conjecture 1. Let $L$ be an alternating transverse link. Then the filtration level of $c\left(\xi_{L}\right) \in \widehat{H F}(-\Sigma(L))$ is equal to the homological degree of $\psi(L)$, and $\psi(L)$ is mapped to $c_{0}\left(\xi_{L}\right)$ under the isomorphism between $\overline{K h}(L)$ and the associated graded group of $\widehat{H F}(-\Sigma(L))$.

Remark 5. Strictly speaking, the above isomorphism depends on the link diagram: while the spectral sequence of [OS2] is believed to be a link invariant, this has not been established. Therefore, we have to fix a braid diagram for our considerations.

In [Pl2], we prove a special case of conjecture 1.

Theorem 5. Let $L$ be a transverse link represented by a closed braid whose diagram is alternating. Then the filtration level of $c\left(\xi_{L}\right)$ is 0 , and $\psi(L)=c_{0}\left(\xi_{L}\right)$.

It should be said that the class of alternating braids is very narrow, and that $\psi(L)=c\left(\xi_{L}\right)=0$ for most of them.

\section{Acknowledgements}

I would like to thank Jake Rasmussen for extremely helpful email correspondence, and Peter Kronheimer and Ciprian Manolescu for illuminating conversations and encouragement. 


\section{References}

[BN] D. Bar-Natan, On Khovanov's categorification of the Jones polynomial, Algebr. Geom. Topol. 2 (2002), 337-370.

[Be] D. Bennequin, Entrelacements et équations de Pfaff. (French), Third Schnepfenried geometry conference, Vol. 1 (Schnepfenried, 1982), 87-161, Astérisque, 107-108 Soc. Math. France, Paris, 1983.

[Bi] J. Birman, Braids, links and mapping class groups, Annals of Math. Studies 82, Princeton Univ. Press, Princeton, NJ, 1974.

[BM] J. Birman and B. Menasco, Stabilization in the braid groups-II: Transversal simplicity of knots, math.GT/0310280.

[Ch] Yu. Chekanov, Differential algebra of Legendrian links, Invent. Math. 150 (2002), no. 3, 441-48.

[EGH] Ya. Eliashberg, A. Givental, and H. Hofer, Introduction to symplectic field theory, Geom. Funct. Anal. (2000), Special Volume, Part II, 560-673.

[Et] J.Etnyre, Legendrian and transversal knots, math.SG/0306256.

[EH1] J. Etnyre and K. Honda, Knots and contact geometry. I. Torus knots and the figure eight knot J. Symplectic Geom. 1 (2001), no. 1, 63-120.

[EH2] — On connected sums and Legendrian knots Adv. Math. 179 (2003), no. 1, 59-74.

[EH3] Cabling and transverse simplicity, math.SG/0306330.

[HO] M. Hedden and P. Ording, The Ozsváth-Szabó and Rasmussen concordance invariants are not equal, math.GT/0512348.

[Ja] M. Jacobsson, An invariant of link cobordisms from Khovanov's homology theory, math.GT/0206303.

[Kh] M. Khovanov, A categorification of the Jones polynomial, Duke Math. J. 101 (2000), no. 3, 359-426.

[KM] P. Kronheimer and T. Mrowka, Gauge theory for embedded surfaces. I, Topology 32 (1993), no. $4,773-826$.

[Lee] E. S. Lee, An endomorphism of the Khovanov invariant, Adv. Math. 197 (2005), no. 2, $554-586$.

[Ng] L. Ng, Computable Legendrian invariants, Topology 42 (2003), no. 1, 55-82.

[OSh] S. Orevkov and V. Shevchishin, Markov theorem for transversal links, J. Knot Theory Ramifications 12 (2003), no. 7, 905-913.

[OS1] P. Ozsváth and Z. Szabó, Knot Floer homology and the four-ball genus, Geom. Topol. 7 (2003), 615-639.

[OS2] On the Heegaard Floer homology of branched double-covers, Adv. Math. 194 (2005), no. 1, 1-33.

[P11] O. Plamenevskaya, Bounds for the Thurston-Bennequin number from Floer homology, Algebr. Geom. Topol. 4 (2004), 399-406.

[P12] , Transverse knots and Heegaard Floer contact invariants for branched double covers. Preprint.

[Ra] J. Rasmussen, Khovanov homology and the slice genus, math.GT/0402131.

$[\mathrm{Ru}]$ L. Rudolph, Quasipositivity as an obstruction to sliceness, Bull. Amer. Math. Soc. 29 (1993), no. $1,51-59$.

[Sh] A. Shumakovitch, Rasmussen invariant, Slice-Bennequin inequality, and sliceness of knots, math.GT/0411643.

[Wr] N. Wrinkle, The Markov Theorem for transverse knots, math.GT/0202055.

Department of Mathematics, M.I.T., Cambridge, MA 02139

E-mail address: olga@math.mit.edu 